\title{
UNDERSTANDING THE KAUFFMAN BRACKET SKEIN MODULE
}

\author{
DOUG BULLOCK \\ Department of Mathematics, Boise State University, Boise, ID 83725, USA \\ email: bullock@math.idbsu.edu \\ CHARLES FROHMAN \\ Department of Mathematics, University of Iowa, Iowa City, IA 52245, USA \\ email: frohman@math.uiowa.edu \\ JOANNA KANIA-BARTOSZYŃSKA \\ Department of Mathematics, Boise State University, Boise, ID 83725, USA \\ email:kania@math.idbsu.edu
}

\begin{abstract}
The Kauffman bracket skein module $K(M)$ of a 3-manifold $M$ is defined over formal power series in the variable $h$ by letting $A=e^{h / 4}$. For a compact oriented surface $F$, it is shown that $K(F \times I)$ is a quantization of the $S L_{2}(\mathbb{C})$-characters of the fundamental group of $F$, corresponding to a geometrically defined Poisson bracket. Finite type invariants for unoriented knots and links are defined. Topologically free Kauffman bracket modules are shown to generate finite type invariants. It is shown for compact $M$ that $K(M)$ can be generated as a module by cables on a finite set of knots. Moreover, if $M$ contains no incompressible surfaces, the module is finitely generated.

Keywords: Knot, Link, 3-manifold, Skein module, Character theory
\end{abstract}

\section{Introduction}

There are two important classes of invariants of links and manifolds that are clearly related, however, conceptual bridges connecting the two are rather sparse. The first type, classical invariants, are derivable from the representation theory of 3-manifold groups. Examples include the Casson-Walker invariant and the Chern-Simons invariant. Quantum invariants are the other type. Using a difficult axiomatic computation, Murakami showed that if the quantum $S U(2)$ invariants are expanded as power series, then the linear terms recapture the Casson-Walker invariant [20] Witten derived an asymptotic formula, using path integrals, relating quantum invariants to classical invariants. Using tools from classical analysis, Rozansky proved that Witten's formula is essentially correct when the manifolds are Seifert Fibered spaces with 3-singular fibers over the sphere [24]. What is needed is a routine for passing from one kind of information to the other.

In a series of papers, Alekseev, Buffenoir, Grosse, Roche and Schomerus [ [1] $\overline{9}_{1}^{\prime} 1 \overline{1}_{1}^{\prime \prime}$ have approached this problem using tools from lattice gauge field theory. It 
is an easy leap from their work to a construction of a quantization of the $S L_{2}(\mathbb{C})$ characters of a surface group. However, the construction is so abstract that it is difficult to get a handle on what this quantization "looks" like. It seems it should play a central role in the transference of data from the classical setting to the quantum setting. What we propose here, is a natural quantization of the $S L_{2}(\mathbb{C})$ characters of a surface group. Since there are already constructions of quantum

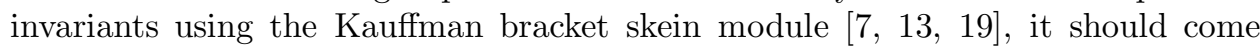
as no surprise that we use it here. The Kauffman bracket skein module of $F \times I$, where $F$ is a compact orientable surface, is a quantization of the the ring of $S L_{2}(\mathbb{C})$ characters of the fundamental group of $F$ with respect to a geometrically defined complex linear Poisson structure.

There is already a small body of literature on skein quantizations of Poisson

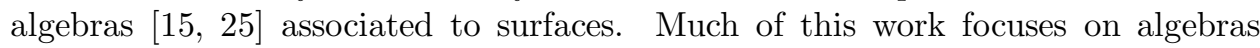
which lack the refined structure of the ring of characters. In particular, one finds quantizations of a symmetric tensor algebra constructed from loops on the surface. These use skein modules other than the Kauffman bracket module, and somewhat different definitions of quantization.

Przytycki was aware that the Kauffman bracket skein module of $F \times I$, defined over Laurent polynomials in the variable A can be viewed as a deformation of the commutative algebra obtained by setting $A=-1$. Furthermore, he knew that this module is free. We show that a completion of it is actually a natural quantization of the $S L_{2}(\mathbb{C})$-characters of a surface group with respect to the standard Poisson structure coming from a bilinear form on $s l_{2}\left[\overline{6} \overline{6}, \overline{1} \overline{1} \overline{4}_{1}\right]$.

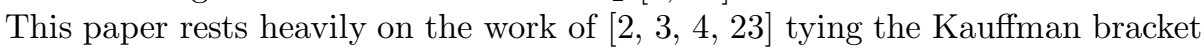
skein module to the ring of $S L_{2}(\mathbb{C})$-characters of the fundamental group of a 3 manifold. We exploit the isomorphism from [in in in order to relate state sum invariants with invariants coming from representation theory. The concept that makes this paper work is redefining the Kauffman bracket skein module over formal power series. This is similar in spirit to Murakami's expansion of $S U(2)$ invariants as power series. In our expansion, the 0 -th order term is the ring of $S L_{2}(\mathbb{C})$-characters. The authors expect that skein modules will become a unifying concept in the theory of invariants of 3-manifolds.

In the next section Poisson rings, quantization, the Kauffman bracket skein module, and the ring $X[M]$ of $S L_{2}(\mathbb{C})$-characters of a manifold $M$ are defined. In the third section a geometric Poisson bracket is defined on the ring of $S L_{2}(\mathbb{C})$ characters of any compact oriented surface $F$. We prove that $K(F \times I)$ forms a quantization of $X[F]$ with respect to that Poisson bracket. Finally, in the last

section we discuss finite type invariants and topological generators. In [22] Przytycki defined finite type invariants in a way similar to ours.

\section{Definitions}

Let $A$ be a commutative algebra with unit over the complex numbers $\mathbb{C}$. A Poisson bracket on $A$ is a bilinear map $\{\}:, A \otimes A \rightarrow A$ that satisfies three conditions.

- The first is antisymmetry, for every $a, b \in A,\{a, b\}=-\{b, a\}$. 


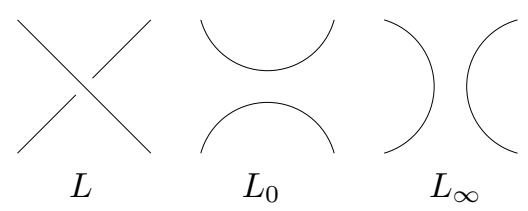

Fig. 1.

- The second is the Jacobi identity, for every $a, b, c \in A$,

$$
\{a,\{b, c\}\}+\{b,\{c, a\}\}+\{c,\{a, b\}\}=0 .
$$

- Finally, the bracket must be a derivation. Hence, for every $a, b, c \in A$,

$$
\{a b, c\}=a\{b, c\}+b\{a, c\} .
$$

A commutative algebra equipped with a Poisson bracket is called a Poisson algebra.

Denote by $\mathbb{C}[[h]]$ the ring of formal power series in $h$, topologized by using the sets $a+h^{n} \mathbb{C}[[h]]$ as a neighborhood basis for the power series $a$. This is known as the $h$-adic topology. We work in the category of topological $\mathbb{C}[[h]]$-modules. If $V$ is a vector space over $\mathbb{C}$, then we can form the module $V[[h]]$ of formal power series with coefficients in $V$ in the obvious way $[1] 6]$. A $\mathbb{C}[[h]]$-module $M$ is topologically free if there exists a vector space $V$ over $\mathbb{C}$ so that $M$ is isomorphic to $V[[h]]$.

If $A$ is a Poisson algebra then, following [i $[1]$, we define a quantization of $A$ to be a $\mathbb{C}[[h]]$-algebra $A_{h}$ together with a $\mathbb{C}$-algebra isomorphism, $\Theta: A_{h} / h A_{h} \rightarrow A$, so that

- as a module $A_{h}$ is topologically free, and

- if $a, b \in A$ and $a^{\prime}, b^{\prime}$ are any elements of $A_{h}$ with $\Theta\left(a^{\prime}\right)=a$ and $\Theta\left(b^{\prime}\right)=b$, then

$$
\Theta\left(\frac{a^{\prime} b^{\prime}-b^{\prime} a^{\prime}}{h}\right)=\{a, b\}
$$

where the equation above implicitly uses the fact that $A_{h}$ is topologically free.

The notion of a skein module was first introduced by Przytycki in [ $\left[2 \overline{1}_{1}^{1}\right]$. Let $M$ be a compact oriented 3-manifold. The Kauffman bracket skein module of $M$ is an algebraic invariant, denoted $K(M)$, which is built from the set $\mathcal{L}_{M}$ of framed links in $M$. By a framed link we mean an embedded collection of annuli considered up to isotopy in $M$, and we include the empty collection $\emptyset$. Three links $L, L_{0}$ and $L_{\infty}$ are said to be Kauffman bracket skein related if they can be embedded identically except in a ball where they appear as shown in Fig. $1_{1}^{1}$ (with the blackboard framing). The notation $L \amalg \bigcirc$ indicates the union of $L$ with an unlinked, 0-framed unknot.

Let $\mathbb{C} \mathcal{L}_{M}$ be the vector space whose basis is $\mathcal{L}_{M}$. As above, $\mathbb{C} \mathcal{L}_{M}[[h]]$ is the $\mathbb{C}[[h]]$-module of power series with coefficients in $\mathbb{C} \mathcal{L}_{M}$. Let $t$ be the formal power series $e^{h / 4}$, and let $s$ be the series $t^{2}$. If $L, L_{0}$ and $L_{\infty}$ are Kauffman bracket skein 
related then $L+t L_{0}+t^{-1} L_{\infty}$ is called a skein relation. For any $L$ in $\mathcal{L}_{M}$ the expression $L \amalg \bigcirc+\left(s+s^{-1}\right) L$ is called a framing relation. Let $S(M)$ be the smallest closed submodule of $\mathbb{C} \mathcal{L}_{M}[[h]]$ containing all possible skein and framing relations. We define $K(M)$ to be the quotient $\mathbb{C} \mathcal{L}_{M}[[h]] / S(M)$.

$K_{0}(M)$ is the quotient $K(M) / h K(M)$. Two links represent the same element in $K_{0}(M)$ if they are homotopic as maps from a disjoint union of circles into $M$. Furthermore, $K_{0}(M)$ is an algebra over $\mathbb{C}$. To define multiplication we begin with elements of $K_{0}(M)$ that can be represented by links, and extend by requiring that multiplication distribute over addition. To multiply the equivalence classes of two links, first perturb them to be disjoint, and then take the class of their union.

Let $M$ be a compact oriented manifold, with or without boundary. The set of $S L_{2}(\mathbb{C})$-representations of the fundamental group of $M$ can be viewed as an affine algebraic set $\mathcal{R}(M)$. Choose a finite set of generators $\left\{\gamma_{i}\right\}_{i=1}^{n}$ and relations $r_{j}$ for the fundamental group of $M$. The set of points in $\prod_{i=1}^{n} S L_{2}(\mathbb{C})$ satisfying the matrix equations given by the $r_{j}$ is in one to one correspondence with the set of representations of $\pi_{1}(M)$ into $S L_{2}(\mathbb{C})$. The sets obtained from different presentations of $\pi_{1}(M)$ are algebraically equivalent [i] $\overline{1}_{1}$. Let $R[M]$ be the coordinate ring of $\mathcal{R}(M)$. Conjugation of representations induces an action on the ring $R[M]$. If $f \in R[M], A \in S L_{2}(\mathbb{C})$ and $\rho: \pi_{1}(M) \rightarrow S L_{2}(\mathbb{C})$ then we define $A$. $f$ to be the function with $A . f(\rho)=f\left(A^{-1} \rho A\right)$. The ring of characters of $M, X[M]$, is the subring of $R[M]$ fixed by this action. The ring $X[M]$ is the coordinate ring of the $S L_{2}(\mathbb{C})$-character set, $\mathcal{X}(M)$.

For each $\gamma \in \pi_{1}(M)$ define a function $\operatorname{tr}_{\gamma}: \mathcal{R}(M) \rightarrow \mathbb{C}$ by $\operatorname{tr}_{\gamma}(\rho)=\operatorname{tr}(\rho(\gamma))$, where $t r$ denotes the standard trace on $S L_{2}(\mathbb{C})$. The algebra $X[M]$ is generated by the functions $t r_{\gamma}$. Trace on $S L_{2}(\mathbb{C})$ satisfies three important identities.

- For any $A, B \in S L_{2}(\mathbb{C}), \operatorname{tr}(A B)=\operatorname{tr}(B A)$.

- For any $A \in S L_{2}(\mathbb{C}), \operatorname{tr}(A)=\operatorname{tr}\left(A^{-1}\right)$.

- For any $A, B \in S L_{2}(\mathbb{C}), \operatorname{tr}(A B)+\operatorname{tr}\left(A B^{-1}\right)=\operatorname{tr}(A) \operatorname{tr}(B)$.

The first property implies that the functions $t r_{\gamma}$ are in the fixed subring of $R[M]$. It also implies that if $\gamma$ and $\delta$ are conjugate elements of $\pi_{1}(M)$ then $t r_{\gamma}=t r_{\delta}$. Since the conjugacy classes of the fundamental group of any space can be identified with free homotopy classes of maps from the circle into that space, we can define $t r_{\gamma}$ even when $\gamma$ is a loop that is not based at the basepoint $x_{0}$ of the fundamental group. To do this, use a path from a point on $\gamma$ back to $x_{0}$ to conjugate $\gamma$ to be based at $x_{0}$, then apply the trace. The second property implies that we don't need to be working with oriented loops; no matter how you orient a loop $\gamma$ you get the same function $t r_{\gamma}$. The third property allows us to define an isomorphism from $K_{0}(M)$ to $X[M]$. If $\Gamma$ is a union of loops $\gamma_{i}, i=1 \ldots n$, then we let $n_{\Gamma}=\prod_{i}-t r_{\gamma_{i}}$. We can define a map

$$
\Theta: K_{0}(M) \rightarrow X[M]
$$

by sending $\Gamma$ to $n_{\Gamma}$ and extending linearly. The map is a well defined surjection of algebras [2] whose kernel is the ideal of nilpotent elements of $K_{0}(M)$.

We restrict our attention to surfaces, where Przytycki and Sikora [23i] have shown that there are no nilpotents. If two loops from a collection $\Gamma$ intersect transversely, 
then we can resolve the intersection via

$$
X=-\leadsto-2 \nearrow
$$

If $\Gamma_{0}$ is the first smoothing and $\Gamma_{\infty}$ is the second, then (2.1) implies

$$
n_{\Gamma}=-n_{\Gamma_{0}}-n_{\Gamma_{\infty}}
$$

in $X[M]$. This allows us to give a state sum model for rewriting polynomials in the traces of curves in terms of polynomials in the traces of curves without intersections. Suppose that there are $k$ crossings in $\Gamma$ that you want to remove. There are two smoothings at each crossing, hence, $2^{k}$ different ways of smoothing all the crossings. We call each of these a state. It is easy to see that

$$
n_{\Gamma}=(-1)^{k} \sum_{\text {states } S} n_{S}
$$

\section{The Kauffman Bracket Module as a Quantization}

We begin with some reminders that will help the reader understand where the Poisson bracket on the $S L_{2}(\mathbb{C})$-characters of a surface group comes from. The standard basis for the Lie algebra $s l_{2}$ is given by

$$
X=\left(\begin{array}{ll}
0 & 1 \\
0 & 0
\end{array}\right), \quad H=\left(\begin{array}{cc}
1 & 0 \\
0 & -1
\end{array}\right) \quad \text { and } \quad Y=\left(\begin{array}{ll}
0 & 0 \\
1 & 0
\end{array}\right) .
$$

We use the nondegenerate $a d$-invariant bilinear form $B: s l_{2} \otimes s l_{2} \rightarrow \mathbb{C}$, which, with respect to the standard basis, is given by

$$
\left(\begin{array}{ccc}
0 & 0 & -1 \\
0 & -2 & 0 \\
-1 & 0 & 0
\end{array}\right) .
$$

It should be noted that $B$ is $\frac{1}{4}$ of the standard Killing form. The form $B$ allows us to identify $s l_{2}^{*}$ and $s l_{2}$. Hence, the complex differential of a holomorphic map $f: S L_{2}(\mathbb{C}) \rightarrow \mathbb{C}$ has a gradient, $\delta_{f}$, with respect to $B$. The gradient is defined by $\delta_{f}(A)=Z$, where $B(Z, W)=d f_{A}(W)$ and the complex tangent space of $S L_{2}(\mathbb{C})$ at $A$ is identified with $s l_{2}$ via left translation.

The gradient of the trace is an interesting example. We begin by computing its differential at the point

$$
A=\left(\begin{array}{ll}
a_{11} & a_{12} \\
a_{21} & a_{22}
\end{array}\right) .
$$

To do this, choose a path $\gamma(h)=A \exp (h X)$ through $A$ in the direction of $X$. Then

$$
d \operatorname{tr}_{A}(X)=\left.\frac{d}{d h} \operatorname{tr}(\gamma(h))\right|_{h=0}=a_{21} .
$$

The values of $d \operatorname{tr}_{A}$ on $Y$ and $H$ are computed by differentiating along the paths $A \exp (h Y)$ and $A \exp (h H)$ respectively. From the above definition of gradient we obtain

$$
\delta_{t r}(A)=-a_{21} Y-a_{12} X-\frac{1}{2}\left(a_{11}-a_{22}\right) H .
$$



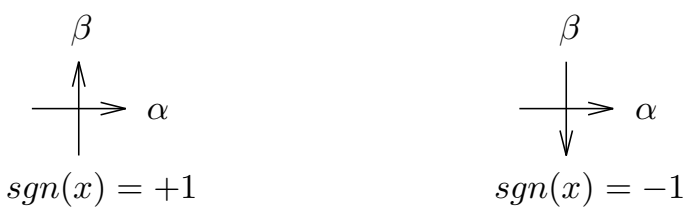

Fig. 2.

It is also worth noting that

$$
B\left(\delta_{t r}(A), \delta_{t r}(B)\right)=\frac{1}{2} \operatorname{tr}\left(A B^{-1}\right)-\frac{1}{2} \operatorname{tr}(A B) .
$$

In $[\overline{1} \mid \overline{1}]$, building on work of $[1 \overline{1} 4]$, it is shown that the coordinate ring of the character variety $\mathcal{X}_{G}(F)$ of the representations of the fundamental group of a compact oriented surface $F$ into a Lie group $G$ admits a Poisson structure. Let $B$ be an $a d$-invariant bilinear form on the Lie algebra $\mathfrak{g}$ of $G$. We use $B$ to define $\delta_{f}: G \rightarrow \mathfrak{g}$ for any $f: G \rightarrow \mathbb{R}$ as above. Let $\gamma$ and $\eta$ be conjugacy classes of $\pi_{1}(F)$. Let $P$ and $Q$ be transverse loops representing $\gamma$ and $\eta$. If $x \in P \cap Q$, define the sign of $x$ via the convention shown in Fig. 2 . For each point $x \in P \cap Q$, choose a path $c_{x}$ joining the basepoint of $\pi_{1}(X)$ to $x$. Let $\gamma_{x}$ be the element of $\pi_{1}(X)$ obtained by first traversing $c_{x}$, then $\gamma$ and then $c_{x}^{-1}$. Define $\eta_{x}$ similarly.

As with $S L_{2}(\mathbb{C})$, we may form the set $\mathcal{R}_{G}(F)$ of representations into $G$. Then $X_{G}[F]$ is, again, the fixed subring of its coordinate ring. If $f: G \rightarrow \mathbb{R}$ is an invariant function on $G$ then we can define $f_{\gamma} \in X[F]$ by letting $f_{\gamma}(\rho)=f(\rho(\gamma))$. According to [i.

$$
\left\{f_{\gamma}, f_{\eta}^{\prime}\right\}(\rho)=\sum_{x \in P \cap Q} \operatorname{sgn}(x) B\left(\delta_{f}\left(\rho\left(\gamma_{x}\right)\right), \delta_{f^{\prime}}\left(\rho\left(\eta_{x}\right)\right)\right) .
$$

We wish to interpret (3.7i) in our own setting. Making the minor adjustment of working complex linearly, we use the previously defined form $B$ on $s l_{2}$. In this case the only invariant function needed is the trace, whose gradient is given in ( $(\overrightarrow{3} . \overline{5})$. For convenience, we allow one symbol to denote both a curve on a surface and its free homotopy class. If $\alpha$ and $\beta$ intersect transversely at a point $x$, define $\alpha \beta_{x}$ to be the loop constructed by first traversing $\alpha$ beginning at $x$ and then traversing $\beta$ (Fig. 김. Using $(3.6)$ we see that

$$
\left\{t r_{\alpha}, t r_{\beta}\right\}=\sum_{x \in \alpha \cap \beta} \operatorname{sgn}(x) \frac{1}{2}\left(t r_{\alpha \beta_{x}^{-1}}-t r_{\alpha \beta_{x}}\right) .
$$

The formula above is well and good, but it could be better. Recalling that the trace of $A$ and the trace of $A^{-1}$ are the same, there should be a formula for

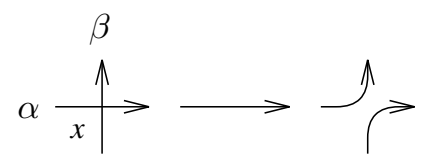

Fig. 3. 

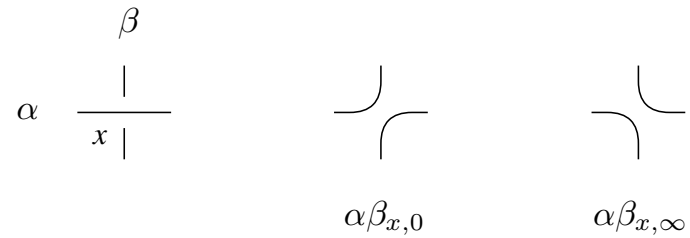

Fig. 4.

the Poisson bracket that does not depend on orientations. Suppose that $\alpha$ and $\beta$ are simple closed curves that intersect one another transversely. It is simpler to use $n_{\gamma}=-t r_{\gamma}$, because the skein rule from the previous section can be used. To represent $\left\{n_{\alpha}, n_{\beta}\right\}$ draw a link $L_{\alpha \cup \beta}$ consisting of $\alpha$ and $\beta$ with $\alpha$ always crossing over $\beta$. At any crossing define two new links, $\alpha \beta_{x, 0}$ and $\alpha \beta_{x, \infty}$ according to the rule in Fig. 14, where you reinterpret the resulting objects as a loop in the surface.

\section{Proposition 1.}

$$
\left\{n_{\alpha}, n_{\beta}\right\}=\sum_{x \in \alpha \cap \beta} \frac{1}{2} n_{\alpha \beta_{x, 0}}-\frac{1}{2} n_{\alpha \beta_{x, \infty}} .
$$

Proof. Compare (3.8i) and (3.9i).

The formula above is still not good enough. We need to have a formula for the Poisson bracket that allows us to compare it to other brackets. Suppose that $\alpha$ and $\beta$ intersect in $k$ points. Each term of the summation for $\left\{n_{\alpha}, n_{\beta}\right\}$ has $k-1$ crossings of $\alpha$ over $\beta$ remaining. There is a state sum model for (3.9) obtained by resolving all these crossings according to Fig. 啇 Notice that each state of this model occurs exactly $k$ times in the resolution. Let $0(S)$ be the number of resolutions of type zero used in making the state $S$, and let $\infty(S)$ be the number of resolutions of type infinity used.

\section{Proposition 2.}

$$
\left\{n_{\alpha}, n_{\beta}\right\}=(-1)^{k} \sum_{\text {states } S} \frac{1}{2}(\infty(S)-0(S)) n_{S} .
$$

Proof. Keep track of the crossings as you resolve.

Define a Poisson structure on $K_{0}(F \times I)$ as follows. If $a$ and $b$ are in $K_{0}(F \times I)$, choose $\bar{a}$ and $\bar{b}$ in $K(F \times I)$ that map down to $a$ and $b$ under the quotient map. Let

$$
\{a, b\}=\frac{\bar{a} \bar{b}-\bar{b} \bar{a}}{h} \quad \bmod h K(F \times I) .
$$

Proposition 3. The isomorphism $\Theta: K_{0}(F \times I) \rightarrow X[F]$ is a morphism of Poisson algebras.

Proof. Compute $\bar{a} \bar{b}-\bar{b} \bar{a}$ by resolving into states corresponding to the crossings of $\bar{a}$ and $\bar{b}$. Since the crossings in the first product are exactly the crossings in the 

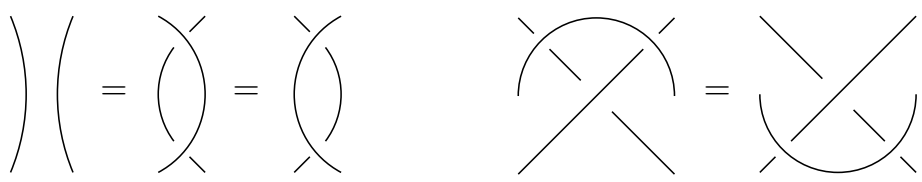

Fig. 5.

second product we get,

$$
\sum_{\text {states } S}\left((-t)^{0(S)-\infty(S)}-(-t)^{\infty(S)-0(S)}\right) S .
$$

Notice that the coefficient of $h$ is

$$
\frac{1}{2}(-1)^{k} \sum_{\text {states } S}(0(S)-\infty(S)) S
$$

where $k$ is the number of times $\bar{a}$ crosses with $\bar{b}$.

If $K(F \times I)$ is topologically free, then $K(F \times I)$ and $\Theta$ form a quantization of $X[F]$. The proof that $K(F \times I)$ is topologically free is an adaptation of ideas of Przytycki [21']. A diagram of a framed link in $F \times I$ is a possibly empty four valent graph in $F$, with overcrossings, undercrossings and blackboard framing. Every framed link can be represented by a diagram, and every diagram represents a framed link. The correspondence is not one-to-one. Two diagrams represent the same framed link if and only if they differ by a sequence of isotopies of $F$ and Reidemeister moves of the second and third types (Fig. $\overline{1}$, Let $\mathcal{D}(F)$ be the module of power series with coefficients in the vector space over the set of diagrams up to isotopy. We define $R S(F)$ to be the smallest closed submodule of $\mathcal{D}(F)$ containing all skein and framing relations along with the two Reidemeister moves admissible for regular isotopy. It is clear that $K(F \times I)$ is the quotient of $\mathcal{D}(F)$ by $R S(F)$.

A trivial circle on a surface is a circle that bounds a disk. Let $\mathcal{B}$ be the vector space over $\mathbb{C}$ whose basis consists of all diagrams that have no crossings and no trivial circles. Let $\mathcal{B}[[h]]$ be the module of formal power series with coefficients in $\mathcal{B}$. Beginning with $\Lambda_{0} \in \mathcal{D}(F)$, construct a sequence as follows. Given $\Lambda_{n-1}$, let $\Lambda_{n-1, n-1}$ be the coefficient of $h^{n-1}$ in $\Lambda_{n-1}$. Resolve the crossings in $\Lambda_{n-1, n-1}$ via skein relations to get an element $B_{n-1}$ of $\mathcal{B}[[h]]$. Let $\Lambda_{n}$ be $\Lambda_{n-1}-\Lambda_{n-1, n-1}+$ $B_{n-1}$. Notice that, by construction, all of the $\Lambda_{n}$ are equivalent modulo $R S(F)$. Furthermore, the sequence is Cauchy and converges to an element of $\mathcal{B}[[h]]$. Define the map $\Psi: \mathcal{D}(F) \rightarrow \mathcal{B}[[h]]$ by letting $\Psi(\Lambda)$ be the limit of this sequence when $\Lambda=\Lambda_{0}$. It is clear that $\Psi$ is well defined and continuous. Hence if $\Psi(R S(F))=\{0\}$ then $\Psi$ descends to a continuous map

$$
\Psi: K(F \times I) \rightarrow \mathcal{B}[[h]]
$$

Since $\Psi$ is linear and continuous, we only need to check this for each of the four relations on a single diagram. The framing relation is trivial. We expand the effect of the second Reidemeister move in Fig. ${ }^{1}{ }_{1}^{6}$. The other two relations work similarly. 


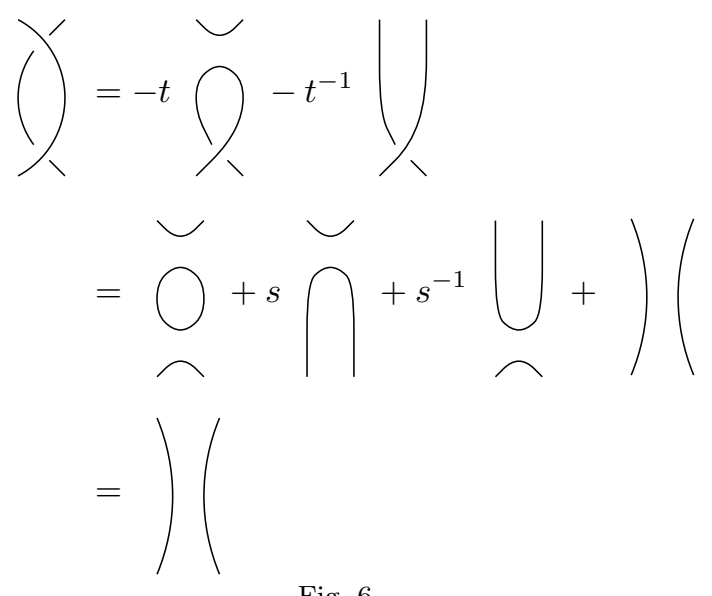

Fig. 6.

From these computations we see that there is a continuous map $\Psi: K(F \times I) \rightarrow$ $\mathcal{B}[[h]]$. There is an obvious map back obtained by including $\mathcal{B}[[h]]$ into $\mathcal{D}(F)$ and then projecting to the quotient. It is easy to check that these maps are the inverses of one another. Therefore the two modules are isomorphic, and we have proved the following theorem.

Theorem 1. The Kauffman bracket skein module $K(F \times I)$ and the map $\Theta: K_{0}(F \times I) \rightarrow X[F]$ form a quantization of $X[F]$.

\section{Applications}

\subsection{Finite type invariants}

One may think of $K(M)$ as a source of invariants of framed, unoriented links in $M$. The invariants are most interesting when the module is topologically free. Suppose there is an isomorphism between $K(M)$ and some $V[[h]]$. Given a framed link $L$ in $M$, take the power series $\sum v_{i} h^{i} \in V[[h]]$ corresponding to the class of $L$ in $K(M)$. The coefficients $v_{i}$ are framed link invariants. By choosing a basis for $V$ we obtain numerical invariants, namely the coefficients of each $v_{i}$ in this basis.

We have seen that $K(F \times I)$ is isomorphic to $V[[h]]$, where $V$ has a natural basis. When $F$ is a disk these invariants are familiar ones. If $L$ is given an orientation then it has a Jones polynomial, $J_{L}$, and a well defined writhe, $w(L)$. Regardless of orientation, $\langle L\rangle=t^{-3 w(L)} J_{L}\left(e^{h}\right)$, provided $J$ is normalized at $J_{\emptyset}=1$. It is well known that the coefficients of $J_{L}\left(e^{h}\right)$ are (oriented) finite type invariants, and that those of $t^{-3 w(L)}$ are (framed and oriented) finite type invariants. It follows that, in some sense, the coefficients of $\langle L\rangle$ are finite type invariants. We introduce one possible definition that makes this precise.

Recursively, a decorated link with $n$ double points represents a linear combination in $\mathbb{C} \mathcal{L}_{M}$. It is a difference of two decorated links with $n-1$ double points as in Fig. $\overline{7}_{\text {- }}$. Here the links are assumed to be identical outside the neighborhood 


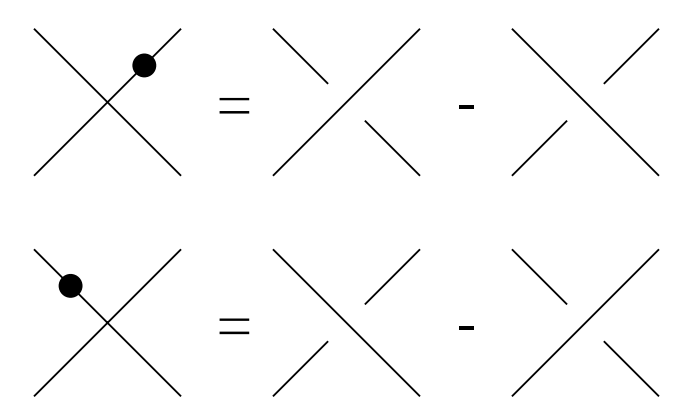

Fig. 7.

shown, and the arcs have a blackboard framing. The dot denotes which of the two possible differences is meant. A choice of dots for the double points of a link is called a decoration.

Noting that a different decoration can only change the linear combination by a multiple of -1 , we define $\mathcal{V}_{n}(M)$ to be the subspace of $\mathbb{C} \mathcal{L}_{M}$ spanned by all decorated links with $n$ double points. Clearly there is a filtration

$$
\mathbb{C} \mathcal{L}_{M}=\mathcal{V}_{0}(M) \supset \mathcal{V}_{1}(M) \supset \cdots \mathcal{V}_{n}(M) \supset \cdots .
$$

A map $f: \mathcal{L}_{M} \rightarrow \mathbb{C}$ is a finite type invariant if, when extended linearly to $\mathbb{C} \mathcal{L}_{M}$, $\mathcal{V}_{n+1}(M)$ lies in its kernel for some $n$. The order of $f$ is the smallest such $n$.

Let $\iota: \mathcal{V}_{0} \rightarrow K(M)$ be the canonical projection.

Proposition 4. $\iota\left(\mathcal{V}_{n}\right) \subset h^{n} K(M)$.

Proof. This is clearly true for $n=0$. If $n>0$ we have

$$
\begin{aligned}
\pm X & =Y-X \\
& =-t)\left(-t^{-1} \smile+t \longleftarrow+t^{-1}\right)( \\
& \left.=\left(t-t^{-1}\right) \longleftarrow-\left(t-t^{-1}\right)\right)(,
\end{aligned}
$$

which implies $\iota\left(V_{n}\right) \subset h \iota\left(V_{n-1}\right)$. The result follows by induction.

Theorem 2. If $K(M)$ is topologically free then the coefficients of a link are finite type invariants. Specifically, let $\Phi: K(M) \rightarrow V[[h]]$ be an isomorphism; let $\left\{b_{\alpha} \mid \alpha \in \mathcal{I}\right\}$ be a basis for $V$; and define $\Phi_{i, \alpha}: K(M) \rightarrow \mathbb{C}$ by

$$
\Phi=\sum_{i}\left(\sum_{\alpha} \Phi_{i, \alpha} b_{\alpha}\right) h^{i} .
$$

For each $i$ and each $\alpha, \mathcal{V}_{i+1}(M)$ lies in the kernel of $\Phi_{i, \alpha} \circ \iota$.

Proof. If $\beta \in \mathcal{V}_{i+1}$ then $\Phi \circ \iota(\beta) \in h^{i+1} K(M)$. Hence $\Phi_{i, \alpha}(\iota(\beta))=0$ for any $\alpha$.

Remark 1. There are several different, though equally reasonable, definitions of finite type invariants of unoriented, framed links. In each case, Proposition 4 and Theorem 2 hold. 


\subsection{Topological Generators}

As an algebra $K(F \times I)$ is generated by a finite set of knots $\left\{K_{1}, \ldots, K_{n}\right\}\left[\begin{array}{ll}50 \\ 0\end{array}\right]$. Ergo, as a module, $K(F \times I)$ is spanned by the set of all products of those generators. Although there is no product structure on $K(M)$ in general, it is possible to capture the geometric essence of these products via the notion of cabling. For a framed knot $K$ in $M$ we define $K^{n}$ to be the framed link formed by taking $n$ parallel copies of $K$, where parallel means each copy is a pushoff of $K$ along its framing. If $n=0$ then $K^{n}=\emptyset$. If $L=K_{1} \cup \cdots \cup K_{m}$ is a framed link, let $L^{\left(n_{1}, \ldots, n_{m}\right)}$ be $K_{1}^{n_{1}} \cup \cdots \cup K_{m}^{n_{m}}$. We will refer to this as the $\left(n_{1}, \ldots, n_{m}\right)$-cable of $L$.

For $L \in \mathcal{L}_{M}$ let $W_{L}$ denote the $\mathbb{C}[[h]]$-linear span of all cables of $L$ in $K(M)$. If $W_{L}$ is dense in $K(M)$ we say that $L$ carries topological generators for $K(M)$. The components of $L$ are the actual generators. A familiar example is $\emptyset$, carrying generators for $K\left(S^{3}\right)$. A non-example is the standard basis of $K(F \times I)$, which consists of framed links that admit projections with no crossings and no trivial components ${ }_{2}^{2} \overline{1}_{1}$. Unless $F$ is planar with three or fewer boundary components, this basis contains infinitely many knot types. Consequently, there is no link whose cables span it.

Theorem 3. Let $K_{1}, \ldots, K_{n}$ be knots that generate $K_{0}(M)$ as an algebra. If $L_{0}$ is a link whose components are $K_{1}, \ldots, K_{n}$, then $L_{0}$ carries topological generators for $K(M)$.

Proof. Consider the inductive hypothesis;

$$
H_{n}: \forall L \in K(M) \exists \beta \in W_{L_{0}} \text { such that } \beta-L \in h^{n} K(M) .
$$

Assume $H_{n}$ for all $i<n$, and choose $L$. As a first step find $\beta_{n-1}$ so that $\beta_{n-1}-L \in$ $h^{n-1} K(M)$. Let $\alpha$ be the constant term of a power series representing $\left(\beta_{n-1}-\right.$ $L) / h^{n-1}$. The idea is to write $\alpha$ as $\sum a_{i} L_{i}$, and resolve each link $L_{i}$ into cables of $L_{0}$. To do this, recall that the knots $\left\{K_{j}\right\}$ generate $K_{0}(M)$. That means there exists $\gamma_{i} \in W_{L_{0}}$ such that $\gamma_{i}-L_{i} \in h K(M)$. It follows that there exists $\beta_{n} \in W_{L_{0}}$, $\beta_{n}+\beta_{n-1}-L \in h^{n} K(M)$. Hence $H_{n}$ is true for all $n$. Since $\left\{h^{n} K(M)\right\}$ is a neighborhood basis for 0 , we have shown that $L$ lies in the closure of $W_{L_{0}}$.

Scholium 1. If $K_{0}(M)$ is finitely generated as a $\mathbb{C}$-module, then $K(M)$ is finitely generated as a $\mathbb{C}[[h]]$-module.

Proof. Let $\alpha_{1}, \ldots, \alpha_{n}$ be elements of $K(M)$ such that $K_{0}(M)$ is generated by their images under the canonical projection. Modify the inductive hypothesis of the preceding proof to read:

$$
H_{n}: \forall L \in K(M) \exists \beta \in \operatorname{span}\left(\left\{\alpha_{i}\right\} \text { such that } \beta-L \in h^{n} K(M) .\right.
$$

The proof that $H_{n}$ holds for all $n$ is essentially the same as above.

Corollary 1. If $K_{0}(M)$ has no nilpotent elements then $X[M]$ induces topological generators for $K(M)$. More specifically, there exists a finite set $t r_{\gamma_{1}}, \ldots, t r_{\gamma_{n}}$ which generates $X[M]$ as a ring. If $K_{1}, \ldots, K_{n}$ are knots chosen to correspond to each $t r_{\gamma_{i}}$, then they are topological generators of $K(M)$.

Recall that a 3-manifold is small if it contains no incompressible surface. 
Theorem 4. If $M$ is a small 3-manifold, then $K(M)$ is finitely generated as a module.

Proof. By Theorem 11 of [2], $K_{0}(M)$ is a finitely generated module.

It is interesting to compare Theorem 4 to a conjecture of Traczyk [1] $\overline{7}_{1}$, Problem $1.92 \mathrm{H}]$.

Conjecture 1. If $M$ is a simply connected 3-manifold other than $S^{3}$ (possibly with holes), then $S_{2, \infty}(M)$ is infinitely generated.

The module $S_{2, \infty}(M)$ is the Kauffman bracket skein module defined over $\mathbb{Z}\left[A, A^{-1}\right]$. For any 3-manifold $M$, there is a map $S_{2, \infty}(M) \rightarrow K(M)$ obtained by substituting $-t$ for $A$. Is this map injective? If it isn't injective what does its kernel look like?

\section{References}

[1] A. Y. Alekseev, H. Grosse, V. Schomerus Combinatorial guantization of the Hamiltonian Chern-Simons theory I-II, hep-th/9403066 and hep-th/9408097.

[2] D. Bullock, The ring of $S L_{2}(\mathbb{C})$-characters is a skein module, preprint.

[3] D. Bullock, Estimating a skein module with $S L_{2}(\mathbb{C})$-characters, preprint.

[4] D. Bullock, Estimating the states of the Kauffman bracket skein module, preprint.

[5] D. Bullock, A finite set of generators for the Kauffman bracket skein algebra, preprint.

[6] I. Biswas, K. Guruprasad, Principle bundles on open surfaces and invariant functions on Lie groups, Int. J. Math. 4 (1993) 535-544.

[7] C. Blanchet, N. Habegger, G. Masbaum, P. Vogel, Topological quantum field theories from the Kauffman bracket, Topology 31 (1992) 685-699.

[8] E. Buffenoir, Ph. Roche, Two dimensional lattice gauge theory based on a quantum group , Comm. Math. Phys. 170 (1995)

[9] E. Buffenoir, Ph. Roche, Link invariants and combinatorial quantization of Hamiltonian Chern-Simons theory, '́⿴囗十-alg/9507001!.

[10] E. Buffenoir, Chern-Simons theory on a lattice and a new description of 3-manifold invariants, 'a-alg/9509020.

[11] M. Culler, P. Shalen, Varieties of group representations and splittings of 3-manifolds, Ann. Math. 117 (1983) 109-146.

[12] V. V. Fock and A. A. Rosly, Flat connections and polyubles, Teoreticheskaya i Matematicheskaya Fizika, 95 no. 2, 228-238.

[13] R. Gelca, Topological quantum field theory with corners based on the Kauffman bracket, preprint.

[14] W. Goldman, Invariant functions on Lie groups and Hamiltonian flows of surface group representations, Invent. Math. 85 (1986) 263-302.

[15] J. Hoste, J. H. Przytycki, Homotopy skein modules of orientable 3-manifolds, Math. Proc. Camb. Phil. Soc. 108 (1990), 475-488.

[16] C. Kassel, Quantum groups, Springer-Verlag (1995).

[17] R. Kirby, Problems in low-dimensional topology, preprint

[18] L. Korgodsky, Y. Soibelman, Algebras of functions on quantum groups, preprint.

[19] W. B. R. Lickorish, The skein method for 3-manifold invariants, J. Knot Theory Ramifications 2 no. 2 (1993) 171-194. 
[20] H. Murakami, Quantum SU(2)-invariants dominate Casson's SU(2)-invariant, Math. Proc. Camb. Phil. Soc. 115 (1993) 253-281.

[21] J. H. Przytycki, Skein modules of 3-manifolds, Bull. Polish Acad. Sci. 39 (1991) 91100.

[22] J. H. Przytycki, Vassiliev-Gusarov skein modules of 3-manifolds and criteria for knots' periodicity, preprint

[23] J. H. Przytycki, A. Sikora, private communication.

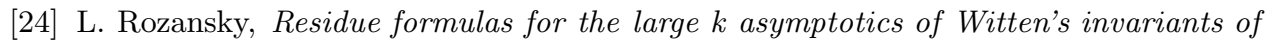
Seifert manifolds: the case of $S U(2)$, hep-th/9412075.

[25] V. G. Turaev, Skein Quantization of Poisson Algebras of Loops on Surfaces, Ann. Scient. Ec. Norm. Sup. 24 (1991) 635-704. 